\title{
The anticodon is the signal sequence for mitochondrial import of glutamine tRNA in Tetrahymena
}

\author{
Christopher P. Rusconi' ${ }^{1}$ and Thomas R. Cech $^{2}$ \\ Department of Molecular, Cellular, and Developmental Biology, Department of Chemistry and Biochemistry, Howard \\ Hughes Medical Institute, University of Colorado, Boulder, Colorado, 80309-0215 USA
}

\begin{abstract}
The import of nuclear-encoded RNAs into mitochondria is required for proper mitochondrial function in most organisms. However, the mechanisms used to achieve RNA import are largely unknown. In particular, the RNA elements that direct import have not been identified in any organism. In Tetrahymena, only one of three nuclear-encoded glutamine accepting tRNAs is imported into mitochondria. We transform Tetrahymena with marked glutamine tRNAs and quantitate their level of accumulation in mitochondria. Of several isostructural nucleotide substitutions tested, alteration of the anticodon sequence uniquely abolishes import. Furthermore, substitution of a single anticodon nucleotide (UUA $\rightarrow$ UUG) confers import on a normally nonimported glutamine tRNA. Thus, the anticodon functions as a mitochondrial localization signal and is both necessary and sufficient for tRNA import. Given the prior evidence that neither the cytoplasmic nor the mitochondrial glutaminyl-tRNA synthetase distinguishes between the imported and nonimported glutamine tRNAs with respect to aminoacylation, we propose that some mitochondrial import factor distinct from a synthetase recognizes the anticodon of the imported glutamine tRNA.
\end{abstract}

[Key Words: RNA import; RNA localization; tRNA structure; Tetrahymena transformation; mitochondria]

Received July 24, 1996; revised version accepted September 27, 1996.

Structural RNAs encoded in the nucleus are critical to mitochondrial function in a wide variety of organisms. Mitochondrial translation in Tetrahymena (Suyama 1967; Chiu et al. 1975), in higher plants (MaréchalDrouard et al. 1988) and in trypanosomatids (Simpson et al. 1989; Hancock and Hajduk 1990) requires the import of nuclear-encoded tRNAs. The nuclear-encoded mitochondrial RNA processing (MRP) RNA of the nuclease MRP appears to play a role in mitochondrial DNA replication in animals and yeast (Chang and Clayton 1987; Schmitt and Clayton 1992; Li et al. 1994). Also, a single nuclear-encoded lysine tRNA is present in the mitochondria of yeast, but its function is unknown (Martin et al. 1979).

Despite the growing number of examples of imported RNAs, very little is known about how RNAs are localized to the mitochondria. RNA import appears to be a very specific process, as examples in which only one of several nuclear-encoded isoaccepting tRNAs is imported have been described in yeast (Martin et al. 1979), Leishmania (Lye et al. 1993; Shi et al. 1994), and Tetrahymena (Rusconi and Cech 1996). Nonetheless, the nature of the

${ }^{1}$ Present address: Department of Experimental Surgery, Duke University Medical Center, Durham, North Carolina 27710 USA.

${ }^{2}$ Corresponding author. information required for import remains largely unknown. While it seems likely that specific cis-acting sequences act as determinants that direct import or as antideterminants that prevent import, sequences directing mitochondrial localization have not been identified in any RNA molecule.

As a first step toward understanding RNA import, we wanted to define the information required for tRNA import in Tetrahymena thermophila. In Tetrahymena, approximately two-thirds of the tRNAs that function in mitochondrial translation appear to be imported (Suyama and Hamada 1976; Suyama 1986). Previously, we have shown that only one of the three glutamineaccepting tRNAs of Tetrahymena is imported into mitochondria (Rusconi and Cech 1996). This imported tRNA, tRNA ${ }^{\text {Gln }}$ (UUG) (which will subsequently be referred to as the imported glutamine tRNA), decodes the normal glutamine codons CAA and CAG used in both mitochondrial and cytosolic translation. Its cellular location is mainly cytosolic, with $\sim 10 \%$ of it localized to the mitochondria. The two nonimported glutamine tRNAs, tRNA ${ }^{\text {Gln }}$ (UUA) and tRNA ${ }^{\mathrm{Gln}}$ (CUA) (which will subsequently be referred to as the nonimported glutamine tRNAs), decode the codons UAA and UAG that are termination codons in the "universal" genetic code but specify glutamine in Tetrahymena cytosolic translation. These tRNAs are exclusively cytosolic, as expected 
because they would suppress normal translation termination in the mitochondrion. When marked versions of the imported and the nonimported glutamine tRNA (with the UUA anticodon) flanked by identical sequences are expressed in Tetrahymena, only the former undergoes mitochondrial import; these results suggested that determinants residing within the imported glutamine tRNA specify its mitochondrial localization (Rusconi and Cech 1996). Comparison of the sequence of the imported glutamine tRNA with those of the two nonimported glutamine tRNAs led us to propose several sequences as candidates for these import determinants.

Using a molecular genetic analysis of tRNA import, we have now determined that the anticodon of the imported glutamine tRNA is necessary for import. This sequence functions as a determinant for import, as alteration to any other sequence abolishes glutamine tRNA import. In addition, the anticodon of the imported glutamine tRNA can serve as a mitochondrial localization signal, as it is capable of directing the import of the nonimported glutamine tRNA.

\section{Results}

\section{Design of mutant $t$ RNAs}

A small number of sequence differences within the glutamine tRNAs covary with import, and these were strong candidates for glutamine tRNA import determi-

A

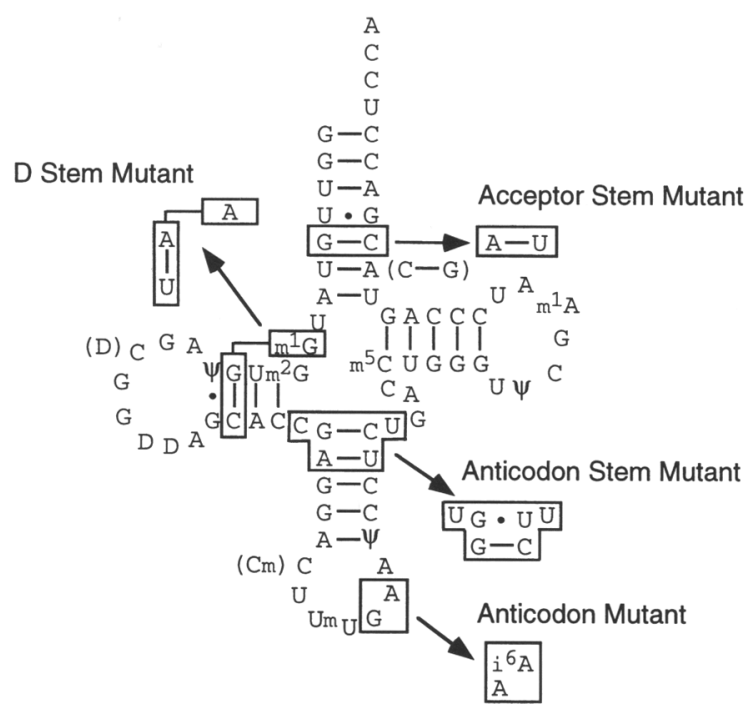

nants (Rusconi and Cech 1996). To determine which of these sequences, or which combination of these sequences, was critical for import, the imported glutamine tRNA sequences were replaced with the corresponding nonimported glutamine tRNA sequences.

Nuclear export of tRNA requires that the tRNA adopt the correct tertiary structure (Tobian et al. 1985). Therefore, to avoid disruption of the tertiary structure of the imported glutamine tRNA during mutational analysis, the sequences that differ between the imported and nonimported glutamine tRNAs were substituted as structural blocks (boxed in Fig. 1A). These candidate tRNA import determinants are (1) the 5.68 base pair of the acceptor stem, $(2)$ the $(12.23) \cdot 9$ base triple of the D stem, (3) the 26.44 tertiary base-base interaction and the 27.43 and 28.42 base pairs at the top of the anticodon stem, and (4) the third base of the anticodon, position 36, which also affects the modification state of position 37 . [Based upon the recognition elements of the $N^{6}$-isopentenyltransferase, if the sequence of the third base of the anticodon (position 36) is anything other than A, position 37 should remain unmodified as in the imported glutamine tRNA (Tsang et al. 1983).] The mutant imported glutamine tRNAs that contain nonimported glutamine tRNA sequences at the above positions will be referred to as the acceptor stem, D stem, anticodon stem, and anticodon mutants, respectively. As shown in Figure 1B these sets of mutations form discrete patches within tRNA, supporting the rationale for grouping them in this manner.

B

Figure 1. Candidates for RNA import determinants within the glutamine tRNAs. $(A)$ Sequence of the imported glutamine tRNA represented as a cloverleaf secondary structure highlighting the sequences that covary with import. The sequences that covary with import have been grouped as structural and/or functional units, and are boxed. Arrows point to sequences found in corresponding positions in the nonimported glutamine tRNAs. Sequences in parentheses are unique to the nonimported glutamine tRNA with the anticodon UUA. All other sequences are identical in the three glutamine tRNAs. $(B)$ Overlay of the import determinant candidates on the three-dimensional structure of yeast tRNA ${ }^{\text {Asp }}$ (Dumas et al. 1985; Westhof et al. 1985), which contains the same number of loop nucleotides as the Tetrahymena glutamine tRNAs. 
The anticodon of the imported glutamine tRNA is critical for mitochondrial localization

Cell lines expressing the acceptor stem, D stem, anticodon stem, and anticodon mutant imported tRNAs were constructed by nuclear transformation of Tetrahymena, followed by screening of cell lines for those expressing the appropriate mutant tRNA. Each of the mutants had its D loop marked by the insertion of two adenines, which allowed direct comparison of the import level of the mutant tRNA with that of the endogenous imported glutamine tRNA by gel electrophoresis and RNA blotting; the D-loop insertion previously had been shown not to affect tRNA import (Rusconi and Cech 1996). If a mutation has no effect on import, then the ratio of the mutant to the endogenous imported glutamine tRNA should be the same in whole cell and mitochondrial RNA fractions; that is, the ratio of the relative abundances of these two tRNAs within the mitochondria and the whole cell (termed the import level) should approach 1 . In contrast, if a mutation inhibits import, then the abundance of the mutant in mitochondria would be reduced relative to the endogenous imported glutamine tRNA (both normalized to their abundance in the whole cell), and the import level would approach 0 .

As shown in Figure 2A and Table 1, the acceptor stem, $\mathrm{D}$ stem, and anticodon stem mutations do not affect import. The abundance of these tRNAs relative to the imported tRNA was the same in mitochondrial and wholecell RNA fractions, and the import level of each was approximately 1 , ranging from 0.8 for the anticodon stem mutant to 1.1 for the D stem mutant. These import levels were essentially identical to the import level of the wild-type marked imported glutamine tRNA /see below and Rusconi and Cech 1996), further indicating that these mutations do not affect import. In contrast, the anticodon mutation, which changes only a single nucleotide within the molecule, abolishes import. The abundance of this tRNA relative to the imported tRNA was dramatically lower in mitochondrial RNA fractions than in whole-cell RNA fractions. Its import level was 0.03 funcorrected for contaminating cytosolic RNA in the mitochondrial preparation), which was quantitatively similar to that of the marked nonimported glutamine tRNA (see below), indicating that its import was completely inhibited by this mutation.

To verify that the import level can legitimately be compared among cell lines, two criteria must be met. First, the amount of endogenous imported glutamine tRNA localized to the mitochondria needs to be similar in each cell line. Second, the enrichment of mitochondrial tRNAs relative to cytosolic tRNAs has to be good for each mitochondrial RNA preparation. The amount of endogenous imported glutamine tRNA localized to mitochondria was determined by measuring its abundance relative to the mitochondrion-encoded tRNA ${ }_{i}{ }^{\text {Met }}$ in mitochondrial RNA fractions as compared with the relative abundance of these two tRNAs in whole-cell RNA fractions. Previously, we determined that $\sim 10 \%$ of the im-
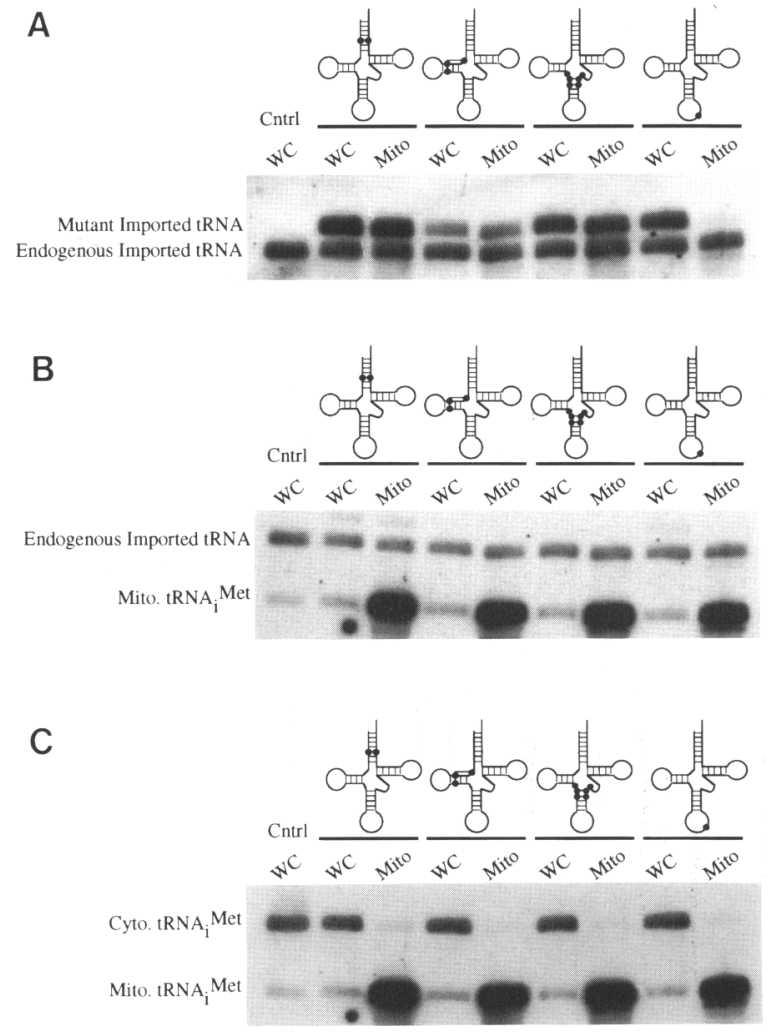

Figure 2. The anticodon of the glutamine tRNA is critical for import. (A) RNA blot analysis of the import level of imported glutamine tRNA mutants. Whole-cell RNA from a cell line transformed with the vector ptRNAexp (Cntrl) and whole cell (WC) and mitochondrial RNAs (Mito) from cell lines expressing D loop-marked imported glutamine tRNAs where different sequences were replaced with the corresponding sequence from nonimported glutamine tRNAs were electrophoresed and electroblotted onto nylon membranes. Positions of the mutations within the imported glutamine tRNA mutants expressed in each cell line are depicted in the tRNA icons above each set of lanes. The amount of whole-cell vs. mitochondrial RNA loaded was such that the signal for the endogenous imported glutamine tRNA was consistent from lane to lane. RNAs were detected by simultaneous hybridization to the ${ }^{32}$ P-labeled oligonucleotide probes Anticodon stem Det, tRNA ${ }^{\text {Gin }}$ (UUG)D, D-Stem Det, tRNA $^{\text {Gln }}$ (UUG) 24-43 and tRNA ${ }^{\mathrm{Gln}}$ (UUG) at a specific activity ratio of $0.6: 1: 1: 1: 1$. This ratio was empirically determined, and normalized the signal of the mutant and endogenous glutamine tRNA in the whole-cell RNA fractions to facilitate direct comparison of their import levels. (B) Control showing that the mitochondrial abundance of the endogenous imported glutamine tRNA is constant in the different cell lines. The RNA blot shown in $A$ was stripped and reprobed simultaneously with the ${ }^{32}$ P-labeled oligonucleotide probes tRNA ${ }^{\mathrm{Gln}}(\mathrm{UUG})$ and mito. $\mathrm{tRNA}_{\mathrm{i}}{ }^{\mathrm{Met}}$ at a specific activity ratio of $1: 0.3$. (C) Control showing that the enrichment for mitochondrial tRNAs in mitochondrial RNA preparations is similar in the different cell lines. The RNA blot shown in $B$ was stripped and reprobed simultaneously with the ${ }^{32} \mathrm{P}$-labeled oligonucleotide probes cyto. tRNA ${ }_{i}{ }^{\text {Met }}$ and mito. RNA $_{i}{ }^{\text {Met }}$ at a specific activity ratio of 1:0.5.

ported glutamine tRNA was localized to the mitochondria of cells analyzed by this method (Rusconi and Cech 1996). As shown in Figure 2B and Table 1, the import 
Table 1. Import of $t R N A^{G I n}(U U G)$ mutants in which sequences are replaced by nonimported $t R N A$ sequences

\begin{tabular}{|c|c|c|c|}
\hline tRNA & $\begin{array}{l}\text { tRNA import } \\
(\text { rel. to } w t)^{a}\end{array}$ & $\begin{array}{l}\text { Percent } \\
\text { endogenous } \\
\text { tRNA }^{\text {Gln }} \\
\text { imported }^{\mathrm{b}}\end{array}$ & $\begin{array}{l}\text { Mitochondrial } \\
\text { tRNA } \\
\text { enrichment }^{\mathrm{c}}\end{array}$ \\
\hline Acceptor stem & $0.9=$ & $4.0 \pm$ & 15 \\
\hline D-stem & $1.1 \pm 0.1$ & $6.7 \pm 0.3$ & $188 \pm 13$ \\
\hline Anticodon stem & $0.8 \pm 0.03$ & $6.5 \pm 0.2$ & $160 \pm 30$ \\
\hline Anticodon & $0.03 \pm 0.02$ & $8.0 \pm 0.2$ & $175 \pm 20$ \\
\hline
\end{tabular}

${ }^{a}$ tRNA import measures the amount of the mutant tRNA imported relative to the endogenous imported glutamine tRNA. It is determined by directly comparing the ratio of the two tRNAs in mitochondrial and whole-cell RNA fractions. Data shown have not been corrected for contamination of the mitochondrial RNA fractions by nonmitochondrial RNAs.

${ }^{\text {b}}$ To verify that expression of mutant tRNAs did not alter the import level of the endogenous glutamine tRNA, the percent endogenous glutamine tRNA imported was determined by directly comparing the ratio of the abundance of the imported glutamine tRNA with that of the mitochondrial initiator methionine tRNA in mitochondrial and whole-cell RNA fractions 'The purity of mitochondrial RNA fractions was assessed by measuring the enrichment of mitochondrial tRNAs relative to nonmitochondrial tRNAs in the mitochondrial RNA fractions. This enrichment was determined by directly comparing the ratio of the abundance of the mitochondrial with the cytosolic initiator methionine tRNAs in mitochondrial and whole-cell RNA fractions. Numbers represent the average \pm the range of two indepedent analyses of RNA from the same cell line. The equations used to calculate each of these values are defined in Materials and Methods.

level varied between 4 and $8 \%$, slightly lower than the $\sim 10 \%$ determined previously. It should be noted that the strains of $T$. thermophila and growth conditions of cells used in this set of experiments were different from our prior experiments, as the strains of Tetrahymena and growth conditions used previously are not compatible with the transformation methods used in this work (Rusconi and Cech 1996). The important conclusion for the current study is that the imported glutamine tRNA was localized to the mitochondria at a similar level in cell lines expressing the four different mutant tRNAs. The enrichment of mitochondrial tRNAs in the mitochondrial RNA preparations was determined by measuring the abundance of mitochondrial tRNA ${ }_{i}{ }^{\text {Met }}$ relative to the cytosolic tRNA ${ }_{i}{ }^{\text {Met }}$ in mitochondrial RNA fractions as compared with the relative abundance of the two tRNAs in whole-cell RNA fractions. Typically, the enrichment for mitochondrial tRNAs in mitochondrial RNA fractions isolated by our method is greater than 150-fold (Rusconi and Cech 1996). As shown in Figure $2 \mathrm{C}$ and Table 1, the enrichment for mitochondrial tRNAs was very similar for mitochondrial RNA fractions isolated from each cell line, varying between 160- and 190-fold. Throughout this study, all data from transfor- mant cell lines shown were confirmed by analysis of an independent transformant cell line of each type.

Because the mitochondrial localization of the imported glutamine tRNA was affected only when its anticodon was changed to that of the nonimported glutamine tRNA, we conclude that the proper sequence of the anticodon of the glutamine tRNA is critical for import. Because the acceptor stem, D stem, and anticodon stem mutations had no appreciable effect on import, these sequences appear not to be essential for tRNA import.

The anticodon sequence UUG is a positive determinant of glutamine tRNA import

The experiments presented above demonstrate that the proper anticodon is required for glutamine tRNA import. This requirement could be explained by two very different models. In the first model, the $\mathrm{G}$ at position 36 functions as a positive determinant for import, directing the mitochondrial localization of this glutamine tRNA. In the second model, the $\mathrm{G}$ at position 36 would not direct import; rather, an A at position 36 (perhaps coupled with the subsequent modification of position 37) would function as an antideterminant for import, preventing interaction of the glutamine tRNA with the tRNA import machinery. These two models lead to very different predictions when position 36 of the anticodon of the imported glutamine tRNA is changed to any sequence other than $G$ or $A$. If the $G$ at position 36 were a determinant for import, then changing this position to any sequence other than G should abolish tRNA import. In contrast, if an A at position 36 were an antideterminant for import, then changing this position to any sequence other than A should allow import to occur. In addition, if only position 36 of the anticodon were being recognized as an import determinant by the tRNA import apparatus, then alteration of the other two anticodon positions should not affect import. In contrast, if the entire anticodon were being recognized as an import determinant by the tRNA import apparatus, changing the other two positions of the anticodon would be expected to abolish import as well.

To determine the role of position 36 , as well as the entire anticodon, in glutamine tRNA import, cell lines were constructed that expressed the imported glutamine tRNA with the anticodon UUC and GCG in the context of the D loop marker as described above. [Attempts to construct cell lines that expressed the imported glutamine tRNA with the anticodon UUU were unsuccessful (data not shown).] As shown in Figure 3 and Table 2, alteration of the imported glutamine tRNA anticodon from UUG to UUC or GCG led to the abolishment of import. The mitochondrial level of these mutants was reduced to the baseline level, that is, the same within error as that of the anticodon mutant (anticodon UUA) and the marked nonimported glutamine tRNA (see below); the measured import levels were 0.06 for the UUC anticodon mutant and 0.05 for the GCG anticodon mutant. By comparison, the import level of the marked im- 


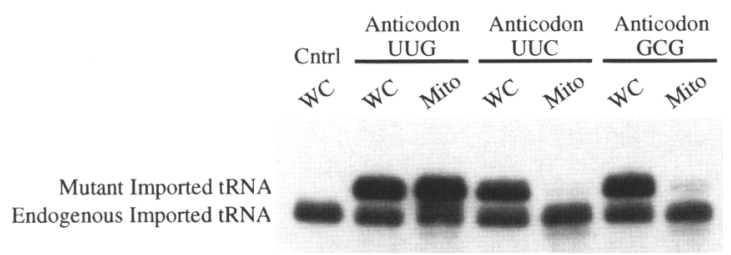

Figure 3. The anticodon of the imported glutamine tRNA is a determinant of tRNA import. Whole-cell RNA from a cell line transformed with the vector ptRNAexp (Cntrl) and whole cell (WC) and mitochondrial RNAs (Mito) from cell lines expressing D loop-marked imported tRNAs with the anticodons UUG (wild type), UUC, or GCG were electrophoresed and electroblotted onto nylon membranes. The amount of whole-cell vs. mitochondrial RNA loaded was such that the signal for the endogenous imported glutamine tRNA was consistent from lane to lane. RNAs were detected by hybridization to the ${ }^{32} \mathrm{P}$ labeled oligonucleotides tRNA ${ }^{\text {Gln }}$ (UUG)D and tRNA ${ }^{\text {Gln }}$ (UUG) $24-43$ at a specific activity ratio of $0.3: 1$. This ratio was empirically determined, and normalizes the signal of the mutant and endogenous imported tRNAs in the whole-cell fraction to facilitate the direct comparison of their import level.

ported glutamine tRNA with the wild-type UUG anticodon was 1.1. As shown in Table 2, the amount of the endogenous imported glutamine tRNA present and the enrichment of mitochondrial tRNAs in each of the mitochondrial RNA fractions were similar for all three of these cell lines and for the cell lines described above, validating the direct comparison of the import level of mutant tRNAs between cell lines.

Because alteration of any base of the anticodon of the imported glutamine tRNA leads to a complete loss of mitochondrial localization, the UUG anticodon is an import determinant necessary to direct glutamine tRNA import.

\section{The anticodon UUG is a glutamine tRNA mitochondrial localization signal}

The data described above demonstrate that the anticodon sequence UUG is a tRNA import determinant, and suggest the possibility that the anticodon of the imported glutamine tRNA may function as a mitochondrial localization signal. By definition, a true mitochondrial localization signal should be capable of localizing a non-

Table 2. The anticodon UUG is necessary for $t R N A^{\text {Gln }}$ import

\begin{tabular}{|c|c|c|c|}
\hline tRNA & $\begin{array}{l}\text { tRNA import } \\
\text { (rel. to wt) }\end{array}$ & $\begin{array}{l}\text { Percent } \\
\text { endogenous } \\
\text { tRNA } \\
\text { imported }\end{array}$ & $\begin{array}{l}\text { Mitochondrial } \\
\text { tRNA } \\
\text { enrichment }\end{array}$ \\
\hline Anticodon UUG & $1.1 \pm 0.05$ & $5.3 \pm 0.3$ & $180 \pm 20$ \\
\hline Anticodon UUC & $0.06 \pm 0.02$ & $4.0 \pm 0.1$ & $185 \pm 30$ \\
\hline Anticodon GCG & $0.05 \pm 0.01$ & $4.4 \pm 0.1$ & $265 \pm 40$ \\
\hline
\end{tabular}

Measurements as defined in Table 1. imported passenger molecule to the mitochondria. To determine whether the anticodon UUG functions as a mitochondrial localization signal, the nonimported glutamine tRNA was used as a test passenger molecule. Cell lines expressing the nonimported glutamine tRNA with the anticodon UUG, UUC, and UUU were constructed in the context of the D loop marked nonimported glutamine tRNA and analyzed as described above. As discussed above for the imported glutamine tRNA, this D loop marker allows direct comparison of the mitochondrial localization of the marked nonimported glutamine tRNA with both the endogenous imported and nonimported glutamine tRNAs, and does not affect the localization of the tRNA molecule (Rusconi and Cech 1996).

As shown in Figure 4A and Table 3, the anticodon UUG was uniquely capable of directing import of the nonimported glutamine tRNA passenger molecule. Nonimported glutamine tRNAs with the anticodon UUG were imported at a level $\sim 60 \%$ of that of the endogenous imported glutamine tRNA. In contrast, nonimported glutamine tRNAs with the anticodon UUA (wild-type), UUC, or UUU were not imported at a level above background. As shown in Table 3, the amount of the endogenous glutamine tRNA imported and the enrichment of mitochondrial tRNAs in the mitochondrial RNA preparations was as described above for the other cell lines.

To further verify that the alteration of a single nucleotide within the nonimported glutamine tRNA had changed its localization from cytosolic only to cytosolic and mitochondrial, the localization of the mutant nonimported glutamine tRNAs was compared with that of the endogenous nonimported glutamine tRNA. As shown in Figure $4 \mathrm{~B}$, marked nonimported glutamine tRNAs with the anticodon UUA, UUC, and UUU were, like the endogenous nonimported glutamine tRNA, barely or not detectable in mitochondrial RNA fractions. In contrast, the nonimported glutamine tRNA with the anticodon UUG was easily detectable in mitochondrial RNA fractions in which the endogenous nonimported glutamine tRNA was undetectable.

The ability of the anticodon UUG to confer import upon the nonimported glutamine tRNA demonstrates that it is sufficient for glutamine tRNA import and that the anticodon of the tRNA functions as a true mitochondrial localization signal.

\section{Discussion}

The nature of the information required for mitochondrial RNA localization has been largely undefined. In general, the molecular identities of import elements are unknown, as are the mechanisms by which the observed specificity of RNA import is generated. In Tetrahymena, only one of three nuclear-encoded glutamine tRNAs is imported into mitochondria (Rusconi and Cech 1996). In this paper, we have shown that the tRNA import apparatus discriminates between these three glutamine tRNAs based on their anticodon sequence. The anticodon functions as a positive determinant for glutamine 


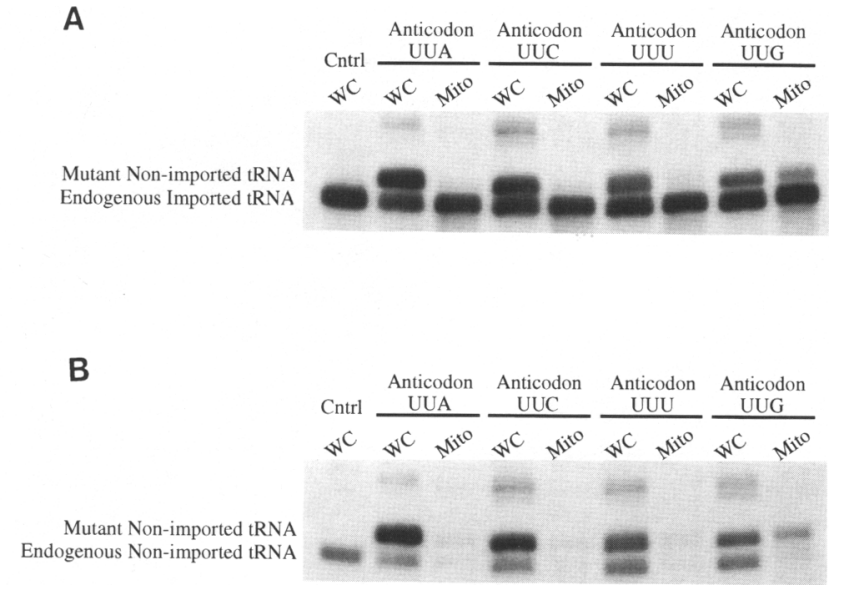

Figure 4. The anticodon of the imported glutamine tRNA is a mitochondrial RNA localization signal. (A) Analysis of the import level of D loop-marked nonimported glutamine tRNAs with the anticodons UUA (wild-type), UUC, UUU, or UUG. Whole-cell RNA from a cell line transformed with the vector ptRNAexp (Cntrl) and whole cell (WC) and mitochondrial RNAs (Mito) from the tRNA-expressing cell lines were electrophoresed and electroblotted onto nylon membranes. The amount of RNA loaded was such that the signal for the endogenous glutamine tRNA was consistent from lane to lane. RNAs were detected by hybridization to the ${ }^{32} \mathrm{P}$-labeled oligonucleotide probes tRNA ${ }^{\mathrm{Gln}}(\mathrm{UUA}) \mathrm{D}$ and tRNA ${ }^{\mathrm{Gln}}$ (UUG) $24-43$ at a specific activity ratio of $1: 0.3$. This ratio was empirically determined, and normalizes the signal of the mutant nonimported glutamine tRNAs to that of the endogenous imported glutamine tRNA in the whole-cell RNA fractions to facilitate the direct comparison of their import levels. The higher molecular weight RNAs apparent in the whole-cell RNA fractions from each cell line have a size and hybridization properties consistent with unprocessed precursors of the D loop-marked nonimported glutamine tRNAs. $(B)$ Comparison of the mitochondrial abundance of the nonimported glutamine tRNA anticodon mutants and the endogenous nonimported glutamine tRNA. The RNA blot from $A$ was stripped and reprobed with the ${ }^{32} \mathrm{P}$-labeled oligonucleotide probes tRNA ${ }^{\mathrm{Gln}}$ (UUA)D and tRNA ${ }^{\mathrm{Gln}}$ (UUA) 24 45 at a specific activity ratio of 1:0.7. This ratio was determined empirically, and normalizes the signal of the mutant and endogenous nonimported glutamine tRNAs in whole-cell RNA fractions to facilitate direct comparison of their mitochondrial abundance.

tRNA import. Furthermore, it is also a mitochondrial signal sequence capable of directing the import of a nonimported glutamine tRNA, analogous to the targeting signals that direct proteins into mitochondria. In this sense, the glutamine tRNA anticodon is more akin to the targeting signals present within the mature region of some imported mitochondrial proteins, as opposed to the typical amino-terminal targeting signals that are presequences removed from mitochondrial proteins during import (Pfanner and Neupert 1990).

\section{tRNA import in Tetrahymena}

Replacement of the anticodon of the imported glutamine tRNA with the anticodon of the nonimported glutamine
Table 3. The substitution of the UUG anticodon confers import on $t R N A^{G I n}(U U A)$

\begin{tabular}{|c|c|c|c|}
\hline tRNA & $\begin{array}{l}\text { tRNA } \\
\text { import } \\
\text { (rel. to wt) }\end{array}$ & $\begin{array}{l}\text { Percent } \\
\text { endogenous } \\
\text { tRNA } \\
\text { imported }\end{array}$ & $\begin{array}{l}\text { Mitochondrial } \\
\text { tRNA } \\
\text { enrichment }\end{array}$ \\
\hline Anticodon UUA & $0.06 \pm 0.02$ & $5.4 \pm 0.2$ & $168 \pm 12$ \\
\hline Anticodon UUC & $<0.08$ & $5.6 \pm 0.1$ & $236 \pm 33$ \\
\hline Anticodon UUU & $<0.1$ & $6.7 \pm 0.2$ & $224 \pm 16$ \\
\hline Anticodon UUG & $0.6 \pm 0.05$ & $6.7 \pm 0.2$ & $185 \pm 35$ \\
\hline
\end{tabular}

Measurements as defined in Table 1.

tRNA abolished import, whereas replacement of the other regions that differed between the two classes of tRNA had no effect. This identified the anticodon as containing the information that specified import. However, it did not reveal how the anticodon of the glutamine tRNAs generated specific tRNA import. The anticodon could give rise to specific tRNA import by functioning as a determinant that localized the imported glutamine tRNA to mitochondria, or as an antideterminant that restricted the nonimported glutamine tRNAs to the cytosol. Two lines of evidence demonstrated that the anticodon of the imported glutamine tRNA is an import determinant. First, mutagenesis of the anticodon of the imported tRNA from UUG to UUA, UUC, or GCG abolished its import, demonstrating that this sequence is necessary for glutamine tRNA import. Second, alteration of the anticodon of the nonimported tRNA from UUA to UUU or UUC did not lead to its import, demonstrating that the anticodon sequence UUA is not restricting this tRNA to the cytosol.

In addition to functioning as a determinant for glutamine tRNA import, the anticodon of the imported glutamine tRNA is also a signal sequence for mitochondrial localization. By definition, a mitochondrial localization signal should be capable, when inserted into a passenger molecule that is normally not imported, of causing that molecule to accumulate within mitochondria. Replacement of the anticodon of the nonimported glutamine tRNA with the anticodon of the imported glutamine tRNA caused it to be localized in mitochondria to a level similar to that of the endogenous imported glutamine tRNA. Thus, at least in the context of a tRNA structure, the UUG anticodon is a signal sequence.

That the anticodon of the imported glutamine tRNA would be a mitochondrial localization signal as well as an import determinant was not a foregone conclusion. Analysis of tRNA identity elements has shown that often several sequences are required to specify correct acylation of a given tRNA, and that strong cooperative interactions can occur between these elements to ultimately determine the identity of a tRNA (Normanly and Abelson 1989; Putz et al. 1993; Rogers et al. 1993). Therefore, although replacement of the other candidate import determinants of the imported glutamine tRNA with nonimported glutamine tRNA sequence had little 
or no effect on mitochondrial import, the possibility that these sequences acted synergistically to direct import was a viable hypothesis. It still remains possible that additional import determinants exist that are common to all three glutamine tRNAs, but such elements would provide no discrimination in interactions with the tRNA import machinery.

Most of the imported tRNAs identified in diverse organisms function in cytosolic translation in addition to mitochondrial translation (Suyama and Hamada 1976; Green et al. 1987; Maréchal-Drouard et al. 1988; Hancock and Hajduk 1990; Schneider et al. 1994; Rusconi and Cech 1996). This implies that a branch point exists in most tRNA import pathways at which molecules to be imported are partitioned either to the cytosol for cytosolic translation or to mitochondria for mitochondrial translation. Although all of the tRNA ${ }^{\text {Gln }}(U U G)$ molecules within Tetrahymena contain the anticodon UUG, and thus the import signal, only $\sim 4-10 \%$ are imported into mitochondria. Partitioning of tRNA ${ }^{\mathrm{Gln}}$ (UUG) between mitochondria and cytosol could be effected by a factor present in a limiting amount that specifically recognizes the anticodon of this imported tRNA. Because no tRNA import factors have been identified in Tetrahymena, it is not clear in which cellular compartment such a factor would initiate import. It could first bind to the tRNA in the nucleus, in which case the factor might compete with the standard nuclear tRNA export factors for binding to tRNA ${ }^{\mathrm{Gln}}(\mathrm{UUG})$, or it could first bind to the tRNA in the cytoplasm, in which case the import factor might compete with the cytosolic glutaminyltRNA synthetase and the translation machinery for binding to tRNA ${ }^{\text {Gln }}$ (UUG). The efficiency of this competition would then determine the $4-10 \%$ level of mitochondrial localization.

\section{RNA import signals in other organisms}

Our findings regarding mitochondrial RNA import in Tetrahymena suggest two general pathways for this process. The first category pertains to organisms like Tetrahymena in which a minor subset of nuclear genes is used to encode a given type of imported RNA. The RNAs to be imported then represent a small subset of a larger pool of cytosolic (or nuclear) RNAs that are not localized to the mitochondria. Specific import in these organisms could be achieved parsimoniously if the standard state of the RNA import machinery were to exclude RNA uptake unless the RNA contained an import determinant that directed its uptake. Based on this idea, RNA import determinants should be present within the imported $t R$ NAs of higher plants, the imported lysine tRNA in yeast, all of the imported tRNAs of Tetrahymena, and the MRP RNA.

In the second category, exemplified by trypanosomatid tRNAs, a majority of a given type of gene encodes imported RNAs. Thus, the RNAs that are not imported represent a small subset of a larger cytosolic (or nuclear) pool of similar RNAs that are to be localized to the mitochondria. Specific RNA import could be achieved in these organisms if the standard state of the RNA import machinery were permissive to the uptake of members of the given class of RNA unless they contained an import antideterminant that restricted the RNA to the cytosol. Based on this idea, RNA import antideterminants would be expected to be found within nuclear-encoded nonimported tRNAs of trypanosomatids.

Are there any data that support this conjecture? Evidence for import determinants in animals and yeast comes from the observation that an internal deletion within the MRP RNA inhibits import (Li et al. 1994), suggesting that sequences or structures within this RNA are acting as import determinants. Also, it appears that import of the lysine tRNA in yeast involves both the cytoplasmic and mitochondrial lysine synthetases (Tarassov et al. 1995). Because both of these proteins are specific RNA-binding proteins, it is reasonable to hypothesize that sequences within this RNA are functioning as import determinants. Evidence that specific RNA import in trypanosomatids is achieved by the presence of antideterminants within nonimported tRNAs is less direct, but intriguing. In Trypanosoma brucei, tRNAs from yeast and human as well as mutants of T. bruceiimported tRNAs all exhibit some mitochondrial localization when expressed from nuclear genes (Hauser and Schneider 1995). This led to the conclusion that no specific signals were required to achieve tRNA import in this organism, but that the tRNA structure alone was sufficient to direct import (Hauser and Schneider 1995), a conclusion consistent with the proposed idea. Yet not every nuclear-encoded tRNA is imported in T. brucei (Hancock and Hajduk 1990). Therefore, it is likely that the nonimported tRNAs contain import antideterminants that generate the observed specificity of tRNA import in this organism.

When RNA import elements are identified in nuclearencoded mitochondrial RNAs from other organisms, the validity of the above model for specific RNA import can be tested.

\section{The potential role of aminoacyl tRNA synthetases in tRNA import}

In Tetrahymena, approximately two-thirds of the mitochondrial tRNAs, including tRNAs for 13 different amino acids, appear to be imported and to function in mitochondrial translation (Suyama and Hamada 1976; Suyama 1986). To explain the specific import of only a subset of nuclear-encoded tRNAs, Suyama and Hamada $(1976,1978)$ proposed that imported tRNAs were localized to mitochondria by their cognate amino-acyl tRNA synthetases. In their proposal, the synthetase serves as a transport protein, functioning to carry its cognate tRNA into mitochondria. This proposal has logical appeal for several reasons. First, the specificity of import would be completely explained by the interaction of the synthetase with its cognate tRNA. No other specificity factors or information within the tRNA outside of that used for recognition by the synthetase would be required for import. Second, because the synthetase-tRNA complex 
would enter the mitochondria through interaction of the synthetase with the mitochondrial protein import machinery, no additional RNA specific import machinery would be required. Finally, because the selectivity of import would be mediated by the cognate synthetase, the localization of a given tRNA to mitochondria would be directly linked to its being a substrate for its mitochondrial synthetase and, thereby, to a function in mitochondrial translation.

However, the mechanistic implications of this model are not fully consistent with either our current understanding of the mechanism of protein import into mitochondria or with the typical stoichiometry of a synthetase to its cognate tRNA. Imported proteins are at least partly unfolded during translocation into mitochondria (Pfanner and Neupert 1990; Hartl and Neupert 1990; Schat $z$ and Dobberstein 1996). Such unfolding is not consistent with the maintenance of noncovalent binding of a synthetase to its cognate tRNA, which requires both the protein and tRNA to maintain a specific tertiary structure (Rich and Schimmel 1977; Rould et al. 1989; Sampson et al. 1990). Therefore, the cotransport of tRNA with synthetase is a problematic mechanism for importing tRNAs into mitochondria. Additionally, the coimport of a tRNA with its cognate synthetase would lead to a 1:1 stoichiometry of synthetase to tRNA, assuming a similar stability of both molecules once imported. It is likely that additional mechanisms would be needed to reduce the relative synthetase-to-cognate tRNA ratio for efficient mitochondrial translation.

At least two groups have presented strong but differing data regarding the role of synthetases in tRNA import. For the import of a lysine tRNA in yeast both the cytoplasmic and mitochondrial lysyl-tRNA synthetases are required, but not sufficient (Tarassov et al. 1995). The apparent role of the cytoplasmic lysyl-tRNA synthetase is to acylate this tRNA prior to its import. The exact role of the mitochondrial lysyl-tRNA synthetase in the import of this tRNA is unknown, but it is required both in vitro and in vivo for mitochondrial accumulation. Although these data support some version of the synthetase model for this tRNA, it should be noted that this tRNA is the only imported tRNA in yeast. Furthermore, it does not appear to function in mitochondrial translation: It is not a substrate for the mitochondrial lysyltRNA synthetase; the yeast mitochondrial genome encodes a lysine tRNA that can decode a similar set of lysine codons; and the acylated form of this molecule is stable in isolated yeast mitochondria (Martin et al. 1977, 1979; Tarassov et al. 1995; Entelis et al. 1996). Therefore, the conclusions drawn from these data regarding the role of synthetases in tRNA import may not be generalizable to the import of multiple tRNAs for use in mitochondrial translation. Perhaps more relevant to the general question of the role of synthetases in the import of tRNAs is recent work in T. brucei (Schneider et al. 1994; Hauser and Schneider 1995). Using an in vivo tRNA import assay, these workers found that a mutant tRNA ${ }^{\text {Tyr }}$ containing an unsplicable intron could be imported into mitochondria. Interestingly, although this tRNA was ef- ficiently imported, it could not be acylated by a cognate aminoacyl synthetase. Therefore, at least in this case, tRNA import is not coupled to acylation, providing no support for a model in which the cognate synthetase serves as a carrier protein.

Can the synthetase model explain the specific import of the Tetrahymena tRNA ${ }^{\mathrm{Gln}}$ (UUG) and, thereby, the role of the anticodon UUG in glutamine tRNA import? All three glutamine tRNAs can be acylated equivalently by the Tetrahymena cytoplasmic glutaminyl-tRNA synthetase, as expected for tRNAs that function in cytoplasmic translation (Suyama and Hamada 1976; Hanyu et al. 1986; Schüll and Beier 1994). Therefore, if the specificity of glutamine tRNA import were to be solely explained by the synthetase model, then the mitochondrial glutaminyl-tRNA synthetase would have to be specific for the imported glutamine tRNA. However, this is not the case. The cytoplasmic and mitochondrial glutaminyltRNA synthetases exhibit the same preference for Tetrahymena whole-cell tRNA versus Escherichia coli tRNA as a substrate for glutamine acceptance, and both synthetases can aminoacylate the three glutamine tRNAs to a similar extent (Suyama and Hamada 1976). Thus, it appears unlikely that the specific import of tRNA ${ }^{\text {Gln }}$ (UUG) can be explained solely by the synthetase model. Some other factor is implicated in the import of this glutamine tRNA to distinguish between these three tRNAs based upon their anticodon sequence. This strongly suggests that the role of the anticodon UUG in glutamine tRNA import is independent of its role as an identity element for recognition by the glutaminyltRNA synthetase, and leaves open the possibility that Tetrahymena mitochondria have tRNA import machinery distinct from their protein import machinery.

\section{Materials and methods}

Construction of a $T R N A$ expression cassette

To facilitate the generation of mutant tRNA genes expressible in T. thermophila, a plasmid containing a tRNA expression cassette, ptRNAexp, was constructed from pH4T2neo (Gaertig et al. 1994). ptRNAexp contains tRNA ${ }^{\text {Gln }}$ (UUG) gene 1, including 182 bp of upstream gene flanking sequence and 82 bp of downstream gene flanking sequence (Rusconi and Cech 1996), with the tRNA coding sequence replaced by an excisable BbsI (NEB) cassette. $B b s I$ is a restriction enzyme that directs DNA cleavage outside of its recognition sequence. The $B b s$ I cassette is a 30-bp sequence that contains $B b s I$ sites at each end inverted with respect to one another, such that upon BbsI digestion of ptRNAexp the 30-bp cassette is entirely removed to generate unique four-nucleotide overhangs within the tRNA gene flanking sequence. Subsequently, synthetic tRNA genes with the appropriate overhanging ends can be ligated into the vector without altering any of the coding or noncoding sequence of the gene. The tRNAexp insert was constructed as follows. First, the overlapping oligonucleotides ExpCas $\mathrm{T}$ and ExpCas B were annealed, and a complete double-stranded molecule was synthesized using T4 DNA Polymerase (NEB). The resultant molecule, which contained $140 \mathrm{bp}$ of upstream gene flanking sequence, the $B b s I$ cassette, and $50 \mathrm{bp}$ of downstream sequence, was used as template in a PCR reaction with the primers EC PCR F and EC PCR $\mathrm{R}$ to generate the full-length tRNAexp insert. The 
tRNAexp insert was then ligated into the EcoRI (NEB) and SacI (NEB) sites of pH4T2neo to generate ptRNAexp. The oligodeoxyribonucleotides described above are as follows. ExpCas T: 5'-ACTTTAATTTTAGAAATATTAATTAAATTATAAACAATTAAATAAAAAATAAATTTTAATTTATTTTTATTTTTTAAGTTAAGTTGGATTGTATTTAAAATCAATAAAAAATTTCTAGTT-3'; ExpCas B: 5'-TATTTTGTAAATATTTAATTTTTTTACTTACAAATTTAATAATGATTTAGGTCTTCGGATCCGTTAACAAGCTTGAAGACGTTATGAAATAATGCTTAATAACTAGAAATTTTTTATTGA-3'; EC PCR F: 5'CGGAATTCTTTTATTATTTTATTAACAAAAAAAATTTTAAAATTGCTAAAACTTTAATTTAATTTTAGAAATATTAATTAAA-3'; EC PCR R: 5'-GCGGAGAGCTCATACAACCAACCGTCATGAAATTAGACTCTTTATTTTTGTTTATTTTGTAAATATTTAATTTTTTTAC-3'

\section{Construction of mutant tRNA genes}

tRNA genes were made synthetically on an Applied Biosystems DNA Synthesizer. For each gene top strand and bottom strand oligodeoxyribonucleotides were synthesized, annealed, and ligated into the site generated by excision of the BbsI cassette from ptRNAexp using standard techniques. In addition to the coding sequence of the tRNA gene, each set of oligonucleotides contained 6 bp of upstream gene flanking sequence and 11 bp of downstream gene flanking sequence to replace those removed from ptRNAexp upon excision of the BbsI cassette. Mutant imported glutamine tRNA encoding genes were constructed using the following oligodeoxyribonucleotides. For acceptor stem mutant Acc. Stem T: 5' - CATAACGGTTCTATGGTGTAGCGGTTAAAGCACCGAGGACTTTGAATCCTCTGACCTGGGTTCGAATCCCAGTAGGACCTTTTTTCT-3' and Acc. Stem B: 5'-ATTTAGAAAAAAGGTCCTACTGGGATTCGAACCCAGGTCAGAGGATTCAAAGTCCTCGGTGCTTTAACCGCTACACCATAGAACCGT-3'; for D stem mutant D-Stem T: 5'-CATAACGGTTGTATAGTATAGCGGTTAAAGTACCGAGGACTTTGAATCCTCTGACCTGGGTTCGAATCCCAGTACGACCTTTTTTCT-3' and D-Stem B: 5'-ATTTAGAAAAAAGGTCGTACTGGGATTCGAACCCAGGTCAGAGGATTCAAAGTCCTCGGTACTTTAACCGCTATACTATACAACCGT-3'; for anticodon stem mutant Anti. Stem T: 5'CATAACGGTTGTATGGTGTAGCGGTTAAAGCACTGGGGACTTTGAATCCCTTGACCTGGGTTCGAATCCDA $\overline{\mathrm{G}}$ TACGACCTTTTTTCT-3' and Anti. Stem B: 5'-ATTTAGAAAAAAGGTCGTACTGGGATTCGAACCCAGGTCAAGGGATTCAAAGTCCCCAGTGCTTTAACCGCTACACCATACAACCGT-3'; for anticodon mutant Anticodon T: 5'-CATAACGGTTGTATGGTGTAGCGGTTAAAGCACCGAGGACTTTAAATCCTCTGACCTGGGTTCGAATCCCAGTACGACCTTTTTTCT-3' and Anticodon B: 5'-ATTTAGAAAAAAGGTCGTACTGGGATTCGAACCCAGGTCAGAGGATTTAAAGTCCTCGGTGCTTTAACCGCTACACCATACAAC'GT-3'; for anticodon UUC Anti. Imp. UUC T: 5'-CATAACCGTTGTATGGTGTAGCGGTTAAAGCACCGAGGACTTTCAATCCTCTGACCTGGGTTCGAATCCCAGTACGACCT'TTTTTCT-3' and Anti. Imp. UUC B: 5'-ATTTAGAAAAAAGGTCGTACTGGGATTCGAACCCAGGTCAGAGGATTGAAAGTCCTCGGTGCTTTAACCGCTACACCATACAACCGT-3'; and for Anticodon GCG Anti. GCG T: $5^{\prime}$-CATAACGGTTGTATGGTGTAGCGGTTAAAGCACCGAGGACTGCGAATCCTCTGACCTGGGTTCGAATCCCAGTACGACCTTTTTTCT-3' and Anti. GCG B: 5'-ATTTAGAAAAAAGGTCGTACTGGGATTCGAACCCAGGTCAGAGGATTCGCAGTCCTCGGTGCTTTAACCGCTACACCATACAACCGT-3' Mutant nonimported glutamine tRNA encoding genes were constructed using the following oligodeoxyribonucleotides. For
Anticodon UUG Anti. UUG T: 5'-CATAACGGTTCCATAGTATAGTGGTTAAAGTACTGGGGACTTTGAATCCCTTTGACCTGGGTTCGAATCCCAGTGGGACCTTTTTTCT-3' and Anti. UUG B: $5^{\prime}$-ATTTAGAAAAAAGGTCCCACTGGGATTCGAACCCAGGTCAAGGGATTCAAAGTCCCCAGTACTTTAACCACTATACTATGGAACCGT-3'; for Anticodon UUC Anti. UUC non-imp. T: 5'-CATAACGGTTCCATAGTATAGTGGTTAAAGTACTGGGGACTTTCAATCCCTTGACCTGGGTTCGAATCCCAGTGGGACC̄TTTTTTCT-3' and Anti. UUC non-imp B: 5'-ATTTAGAAAAAAGGTCCCACTGGGATTCGAACCCAGGTCAAGGGATTGAAAGTCCCCAGTACTTTAACCACTATACTATGGAACCGT-3' for Anticodon UUU Anti. UUU T: 5'-CATAACGGTTCCATAGTATAGTGGTTAAAGTACTGGGGACTTTTAATCCCTTGACCTGGGTTCGAATCCCAGTGGGACCTTTTTTCT-3' and Anti. UUU B: 5'-ATTTAGAAAAAAGGTCCCACTGGGATTCGAACCCAGGTCAAGGGATTAAAAGTCCCCAGTACTTTAACCACTATACTATGGAACCGT-3'. The positions altered within each gene are underlined. In addition to the underlined positions, each gene contains a two-nucleotide AA insertion in the $3^{\prime}$ end of the D loop between positions 21 and 22 that serves as a hybridization and mobility marker (Rusconi and Cech 1996).

Construction and analysis of transformant Tetrahymena cell lines

Construction of $T$. thermophila cell lines expressing mutant tRNAs and isolation of whole-cell and mitochondrial RNA fractions from transformant cell lines were performed as described previously (Rusconi and Cech 1996). RNAs were fractionated on long $(41-\mathrm{cm}) 8 \%$ polyacrylamide $8 \mathrm{M}$ urea gels and detected by RNA blotting as described previously (Rusconi and Cech 1996). RNA blots were visualized by autoradiography or on a Molecular Dynamics PhosphorImager. Mutant tRNAs were detected with the following oligodeoxyribonucleotide probes: for the Acceptor Stem and each imported glutamine tRNA anticodon mutant the previously described tRNA ${ }^{\mathrm{Gln}}(\mathrm{UUG}) \mathrm{D}$ probe was used (Rusconi and Cech 1996); for the D-Stem mutant the probe D-stem Det- 5'-CTCGGTACTTTAACCGCTAT-3' was used; for the Anticodon Stem mutant the probe Anticodon Stem Det- 5'-AGGGATTCAAGTCCCCAGTGC-3' was used; for all the nonimported glutamine tRNA mutants the previously described probe tRNA ${ }^{\mathrm{Cln}}(\mathrm{UUA}) \mathrm{D}$ was used (Rusconi and Cech 1996). The endogenous imported glutamine tRNA was detected with the probes tRNA ${ }^{\operatorname{Gln}}$ (UUG) $24-43$ or $\mathrm{tRNA}^{\mathrm{Gln}}$ (UUG) and the endogenous nonimported glutamine tRNA was detected with the probe tRNA ${ }^{\mathrm{Gln}}$ (UUA) $24-45$, as described previously (Rusconi and Cech 1996). The cytosolic and mitochondrial tRNA $_{i}{ }^{\text {Met }}$ s were detected as described previously (Rusconi and Cech 1996).

\section{Quantitation of $t R N A$ import}

To facilitate the direct comparison of the import level of the mutant and endogenous glutamine tRNAs, the amount of RNA loaded per lane was adjusted such that the signal for the endogenous imported glutamine tRNA was the same in whole cell and mitochondrial RNA fractions, and the specific activities of the probes used was adjusted such that the signal for the mutant tRNA was similar to the signal for the endogenous imported or nonimported glutamine tRNA in the whole-cell RNA fraction. Hybridization signals were quantitated on a PhosphorImager. The effect of a mutation on tRNA import was determined by measuring the signal for the mutant tRNA relative to the wildtype imported tRNA in mitochondrial RNA fractions as com- 
pared with these signals in whole-cell RNA fractions according to the following equation:

mutant tRNA import $=$

$\frac{\text { mito. signal } \frac{\text { mutant tRNA }}{\text { imported glutamine tRNA }}}{\text { whole cell signal } \frac{\text { mutant tRNA }}{\text { imported glutamine tRNA }}}$

The level of endogenous glutamine tRNA import was determined by measuring the signal for the endogenous imported glutamine tRNA relative to the mitochondrial tRNA ${ }_{i}{ }^{\text {Met }}$ in mitochondrial RNA fractions as compared with these signals in whole-cell RNA fractions according to the following equation:

$\%$ endog. glutamine tRNA import $=$

$\frac{\text { mito. signal } \frac{\text { imported glutamine tRNA }}{\text { mito. tRNA }{ }_{i}^{\text {Met }}}}{\text { whole cell signal } \frac{\text { imported glutamine tRNA }}{\text { mito. } \text { tRNA }_{i}^{\text {Met }}}} \times 100$

The purity of the mitochondrial tRNA fractions was determined by measuring the signal for the mitochondrial tRNA ${ }_{i}{ }^{\text {Met }}$ relative to the cytosolic tRNA ${ }_{i}{ }^{\text {Met }}$ in mitochondrial RNA fractions as compared with these signals in whole-cell RNA fractions according to the following equation:

mitochondrial tRNA enrichment $=$

$$
\frac{\text { mito. signal } \frac{\text { mito. tRNA }}{\text { cyto. tRNA }{ }_{i}^{\text {Met }}}}{\text { whole cell signal } \frac{\text { mito. tRNA }_{i}^{\text {Met }}}{\text { cyto. tRNA }{ }_{i}^{\text {Met }}}}
$$

All data from transformant cell lines shown were confirmed by analysis of an independent transformant cell line of each type.

\section{Acknowledgments}

We thank Art Zaug for technical assistance and helpful discussions; Joachim Lingner, Lara Weinstein, and Alex Szewczak for helpful discussions and critical reading of the manuscript; and Cheryl Grosshans and Elaine Podell for synthesis of oligonucleotides. We thank Jacek Gaertig, Jody Bowen, and Martin Gorovsky for Tetrahymena strains, transformation vectors, protocols, and helpful discussions regarding Tetrahymena transformation. We also thank Meng-Chao Yao and David Gilley for helpful discussions regarding Tetrahymena transformations. Finally, we would like to thank Olke Uhlenbeck for help and discussion in designing mutant tRNAs. This work was supported by National Institutes of Health grant GM28039 to T.R.C., and is based on earlier work supported under a National Science Foundation Graduate Research Fellowship to C.P.R. T.R.C. is an Investigator of the Howard Hughes Medical Institute and an American Cancer Society Professor. We thank the W.M. Keck Foundation for their generous support of RNA research at the University of Colorado at Boulder.

The publication costs of this article were defrayed in part by payment of page charges. This article must therefore be hereby marked "advertisement" in accordance with 18 USC section 1734 solely to indicate this fact.

\section{References}

Chang, D.D. and D.A. Clayton. 1987. A mammalian mitochondrial RNA processing activity contains nucleus-encoded RNA. Science 235: 1178-1184.

Chiu, N., A. Chiu, and Y. Suyama. 1975. Native and imported transfer RNA in mitochondria. J. Mol. Biol. 99: 37-50.

Dumas, P., J.P. Ebel, R. Giege, D. Moras, J.C. Thierry, and E. Westhof. 1985. Crystal structure of yeast tRNA ${ }^{\text {Asp: }}$ : Atomic coordinates. Biochimie 67: 597-606.

Entelis, N.S., I.A. Krasheninnikov, R.P. Martin, and I.A. Tarassov. 1996. Mitochondrial import of a yeast cytoplasmic tRNA $^{\text {Lys: }}$ Possible roles of aminoacylation and modified nucleosides in subcellular partitioning. FEBS Lett. 384: 38-42.

Gaertig, J., L. Gu, B. Hai, and M.A. Gorovsky. 1994. High frequency vector-mediated transformation and gene replacement in Tetrahymena. Nucleic Acids Res. 22: 5391-5398.

Green, G.A., L. Maréchal, J.-H. Weil, and P. Guillemaut. 1987. A Phaseolus vulgaris mitochondrial tRNA ${ }^{\mathrm{Lcu}}$ is identical to its cytoplasmic counterpart: Sequencing and in vivo transcription of the gene corresponding to the cytoplasmic tR$\mathrm{NA}^{\mathrm{Lcu}}$. Plant Mol. Biol. 10: 13-19.

Hancock, K. and S.L. Hajduk. 1990. The mitochondrial tRNAs of Trypanosoma brucei are nuclear encoded. I. Biol. Chem. 265: 19208-19215.

Hanyu, N., Y. Kuchino, and S. Nishimura. 1986. Dramatic events in ciliate evolution: Alteration of UAA and UAG termination codons to glutamine codons due to anticodon mutations in two Tetrahymena tRNAs ${ }^{\mathrm{CIn}}$. EMBO $J$. 5: 1307-1311.

Hartl, F.-U. and W. Neupert. 1990. Protein sorting to mitochondria: Evolutionary conservations of folding and assembly. Science 247: 930-938.

Hauscr, R. and A. Schneider. 1995. tRNAs are imported into mitochondria of Trypanosoma brucei independently of their genomic context and genetic origin. EMBO /. 14: 4212-4220.

Li, K., C.S. Smagula, W.J. Parsons, J.A. Richardson, M. Gonzalez, H.K. Hagler, and R.S. Williams. 1994. Subcellular partitioning of MRP RNA assessed by ultrastructural and biochemical analysis. J. Cell Biol. 124: 871-882.

Lye, L.F., D.H. Chen, and Y. Suyama. 1993. Selective import of nuclear-encoded tRNAs into mitochondria of the protozoan Leishmania tarentolae. Mol. Biochem. Parasitol. 58: 233245.

Maréchal-Drouard, L., J.-H. Weil, and P. Guillemaut. 1988. Import of several tRNAs from the cytoplasm into the mitochondria in bean Phaseolus vulgaris. Nucleic Acids Res. 16: 4777-4788.

Martin, N.C., M. Rabinowitz, and H. Fukuhara. 1977. Yeast mitochondrial DNA specifies tRNA for 19 amino acids. Deletion mapping of the tRNA genes. Biochemistry 16: 4672 4677.

Martin, R.P., J.-M. Schneller, A.J.C. Stahl, and G. Dirheimer. 1979. Import of nuclear deoxyribonucleic acid coded lysineaccepting transfer ribonucleic acid (anticodon C-U-U) into yeast mitochondria. Biochemistry 18: 4600-4605.

Normanly, J. and J. Abelson. 1989. tRNA identity. Annu. Rev. Biochem. 58: 1029-1049.

Pfanner, N. and W. Neupert. 1990. The mitochondrial protein import apparatus. Annu. Rev. Biochem. 59: 331-353.

Putz, J., J.D. Puglisi, C. Florentz, and R. Giege. 1993. Additive, cooperative and anti-cooperative effects between identity nucleotides of a tRNA. EMBO /. 12: 2949-2957. 
Rich, A. and P.R. Schimmel. 1977. Structural organization of complexes of transfer RNAs with aminoacyl transfer RNA synthetases. Nucleic Acids Res. 4: 1649-1665.

Rogers, M.J., I. Weygand-Durasevic, E. Schwob, J.M. Sherman, K.C. Rogers, T. Adachi, H. Inokuchi, and D. Soll. 1993. Selectivity and specificity in the recognition of tRNA by E. coli glutaminyl-tRNA synthetase. Biochimie 75: 1083-1090.

Rould, M.A., J.J. Perona, D. Söll, and T.A. Steitz. 1989. Structure of E. coli glutaminyl-tRNA synthetase complexed with tRNA(gln) and ATP at $2.8 \AA$ resolution. Science 246: $1135-$ 1142.

Rusconi, C.P. and T.R. Cech. 1996. Mitochondrial import of only one of three nuclear-encoded glutamine tRNAs in Tetrahymena thermophila. EMBO I. 15: 3286-3295.

Sampson, J.R., A.B. DiRenzo, L.S. Behlen, and O.C. Uhlenbeck. 1990. Role of the tertiary nucleotides in the interaction of yeast phenlyalanine tRNA with its cogante synthetase. Biochemistry 29: 2523-2532.

Schatz, G. and B. Dobberstein. 1996. Common principles of protein translocation across membranes. Science 271: 15191526.

Schmitt, M.E. and D.A. Clayton. 1992. Yeast site-specific ribonucleoprotein endoribonuclease MRP contains an RNA component homologous to mammalian RNase MRP RNA and essential for cell viability. Genes \& Dev. 6: 19751985.

Schneider, A., J. Martin, and N. Agabian. 1994. A nuclear-encoded tRNA of Trypanosoma brucei is imported into mitochondria. Mol. Cell. Biol. 14: 2317-2322.

Schüll, C. and H. Beier. 1994. Three Tetrahymena tRNA ${ }^{\mathrm{Gln}}$ isoacceptors as tools for studying unorthodox codon recognition and codon context effects during protein synthesis in vitro. Nucleic Acids Res. 22: 1974-1980.

Shi, X., D.H. Chen, and Y. Suyama. 1994. A nuclear tRNA gene cluster in the protozoan Leishmania tarentolae and differential distribution of nuclear-encoded tRNAs between the cytosol and mitochondria. Mol. Biochem. Parasitol. 65: 2337.

Simpson, A., Y. Suyama, H. Dewes, D.A. Campbell, and L. Simpson. 1989. Kinetoplastid mitochondria contain functional tRNAs which are encoded in nuclear DNA and also contain small minicircle and maxicircle transcripts of unknown function. Nucleic Acids Res. 17: 5427-5445.

Suyama, Y. 1967. The origins of mitochondrial ribonucleic acids in Tetrahymena pyriformis. Biochemistry 6: 2829-2839.

1986. Two dimensional polyacrylamide gel electrophoresis analysis of Tetrahymena mitochondrial tRNAS. Curr. Genet. 10: 411-420.

Suyama, Y. and J. Hamada. 1976. Imported tRNA: Its synthetase as a probable transport protein. In Genetics and biogenesis of chloroplasts and mitochondria (ed. T. Büchner, W. Neupert, W. Sebald, and S. Werner), pp. 763-770. Elsevier/North Holland Biomedical Press, Amsterdam, The Netherlands.

1978. The mitochondrial and cytoplasmic valyl tRNA synthetases in Tetrahymena are indistinguishable. Arch. Biochem. Biophys. 191: 437-443.

Tarassov, I., N. Entelis, and R.P. Martin. 1995. Mitochondrial import of a cytoplasmic lysine-tRNA in yeast is mediated by cooperation of cytoplasmic and mitochondrial lysyl-tRNA synthetases. EMBO T. 14: 3461-3471.

Tobian, J.A., L. Drinkard, and M. Zasloff. 1985. tRNA nuclear transport: Defining the critical regions of human tRNA $_{i}{ }^{\text {Met }}$ by point mutagenesis. Cell 43: 415-422.

Tsang, T.H., M. Buck, and B.N. Ames. 1983. Sequence specificity of tRNA-modifying enzymes. An analysis of 258 tRNA sequences. Biochim. Biophys. Acta 741: 180-196.
Westhof, E., P. Dumas, and D. Moras. 1985. Crystallographic refinement of yeast aspartic acid transfer RNA. I. Mol. Biol. 184: 119-145. 


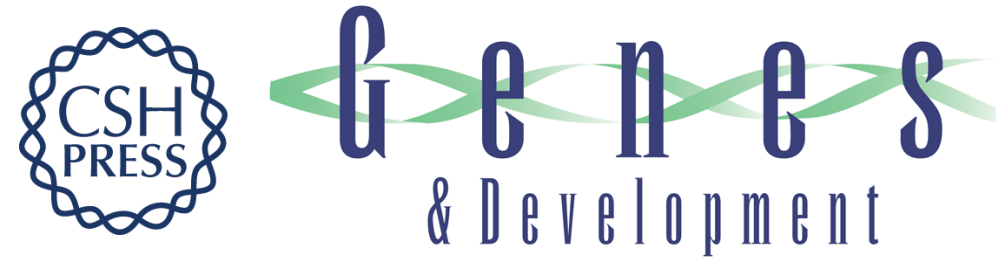

\section{The anticodon is the signal sequence for mitochondrial import of glutamine tRNA in Tetrahymena.}

C P Rusconi and T R Cech

Genes Dev. 1996, 10:

Access the most recent version at doi:10.1101/gad.10.22.2870

References This article cites 36 articles, 8 of which can be accessed free at:

http://genesdev.cshlp.org/content/10/22/2870.full.html\#ref-list-1

License

Email Alerting

Service

Receive free email alerts when new articles cite this article - sign up in the box at the top right corner of the article or click here.

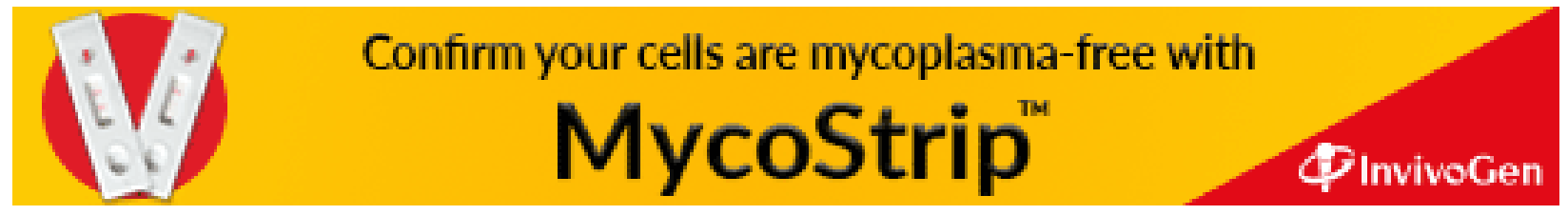

